\title{
A Non Invasive Approach to Prevent Carpel Tunnel Syndrome of IT Women during Pregnancy
}

\author{
C. Nageswari ${ }^{1}$, Dr. N. Meena, ${ }^{2}$ Dr. T. Ramani Devi ${ }^{3}$ \\ ${ }^{1}$ Research Scholar, ${ }^{2}$ Senior Lecturer, ${ }^{3}$ Director Ramakrishna Medical centre \\ ${ }^{1,2}$ Department of Physical Medicine and Rehabilitation, \\ Annamalai University, Annamalainagar, Tamilnadu, India \\ nagesmpt@gmail.com, roshmena@gmail.com, ramanidevidr@yahoo.co.in
}

\begin{abstract}
Carpal Tunnel Syndrome results in considerable discomfort and pain, limitation of activities of daily living, loss of sleep and work disability. Carpal tunnel Syndrome is more frequent in pregnancy because the systemic process increases the extra capsular fluid retention by the hormone Prolactin and produce soft tissue swelling in the later stages (third trimester) of their pregnancies. Many therapies have been advocated for treating the carpal tunnel syndrome including Mobilizations, nerve gliding, tendon gliding, Ultrasound, icing, Massaging, Elevating the arm or flicking, Neural mobilization, wrist splints and invasive treatments like injecting corticosteroids in the carpal tunnel and eventually releasing the median nerve by surgery.etc. Women experience pregnancy several times, and if they acquire CTS, invasive treatments cannot be used, and pregnancy increases the probability of reoccurrence of CTS in the next pregnancy with higher intensity. So it is necessary to use non-invasive treatment methods. The disease is becoming very common nowadays as most of the population is engaged in computer work by some means especially the IT ladies who always use the conventional mouse for their work with the PC. One of the non invasive method of using the wearable device (wearable glove mouse) along with the tendon and nerve gliding exercises prescribed by the physiotherapists improves the condition. The occurrence of CTS is diagnosed by positive Tinel's sign and positive Phalens test. This work tries to give possible non invasive solution for the CTS during pregnancy for IT women professionals and the relief of symptoms is measured with the help of VAS Scale, Functional Status Scale.
\end{abstract}

KEYWORDS: Pregnancy, CTS, IT women, Non invasive Methods, Wearable device, Tendon Gliding, VAS Scale, Functional Status Scale. 


\section{AIM OF THE STUDY}

This study is to aim the effect of to give possible non invasive solution of using the wearable device along with the tendon and nerve gliding exercises for the Carpal tunnel syndrome during pregnancy for IT women professionals to get relief of symptoms.

\section{OBJECTIVE OF THE STUDY}

To determine to prove the effect o non invasive method of using the wearable device (wearable glove mouse) along with the tendon and nerve gliding exercises prescribed by the physiotherapists for improving the condition of carpal tunnel syndrome during pregnancy

\section{METHODOLOGY}

The Randomised Controlled Trait (RCT) is carried in the Department of Physical Medicine and Rehabilitation. Pregnant Women diagnosed with Carpal Tunnel syndrome and are working in IT who are interested in study were invited to undergo Physical examinations. After the confirmation of the CTS with the help of positive Tinel's sign and positive Phalen's test they were separated into two groups. The samples were selected based on inclusion and exclusion criteria. Detailed procedure will be explained in patient's words and informed consent will be obtained from all the participants. The subjects were then allocated into two groups group A (Experimental) and group B (Control).

We have to measure the individual changes, so only two subjects were selected for experimental group who accepts for wearing the glove mouse during her system work along with doing the tendon and nerve gliding exercises. The control group does only exercise program (tendon and nerve gliding exercises). The pregnant women were studied only during the third trimester. Both groups are assessed by outcome measures pre and post interventions.

\section{OUTCOME MEASURES}

Visual Analogue Scale and Functional Status Scale.

\section{STATISTICAL ANALYSIS}

The collected data will be tabulated and analyzed using descriptive \& inferential statistics. To all the parameters mean and standard deviation (SD) will be used. Paired t-test will be used to analyze significant changes between pre-test \& post-test measurements.

\section{RESULTS}

The two-tailed $\mathrm{P}$ value is less than $0.001 \mathrm{By}$ conventional criteria, this difference is considered to be extremely statistically significant and $95 \%$ confidence interval of this difference from the both groups. 


\section{CONCLUSION}

The aim of the study is to give possible non invasive solution of using the wearable device along with the tendon and nerve gliding exercises prescribed by the physiotherapist for the relief of symptoms of Carpal tunnel syndrome during pregnancy period of IT women professionals.

After the treatment sessions the scores on the Visual Analogue Scale and Functional Status Scale indicates that there is decrease of the symptoms of carpal tunnel syndrome during the pregnancy of the IT professionals.

\section{NEED OF THE STUDY}

Carpal tunnel syndrome (CTS) is a frequent complication of pregnancy [1,12]. Most of the studies specifically mentioned the prevalence of CTS in pregnancy is two times higher in third trimester than the first and second trimester of pregnancy. [1,2,3].The true prevalence is unknown, but has been reported to be as high as 62\%.1-6 CTS commonly presents during the third trimester, but can occur during the first trimester. It is the most common compression neuropathy of the upper extremity. Median nerve function is impaired virtually in all pregnant women during the third trimester, even in the absence of symptoms. While most women experience symptomatic improvement following delivery, a significant percentage may still have some complaints and continue to wear splints. A high level of vigilance should be maintained in the management of the pregnant IT women who are working with the computer during pregnancies with the prevalence of CTS. During pregnancy and postpartum period of women the compression neuropathies such as CTS are very common. CTS is seen as a complication of pregnancy [4]. Approximately about $2.3 \%$ to $4.6 \%$ of patients with CTS are pregnant women [5] up to 50\% of all pregnant women have nocturnal hand symptoms, mostly in the third trimester. Hormonal changes and oedema and gestational diabetes are likely the main causes of CTS.[8] After child birth Carpal tunnel syndrome may decrease spontaneously within few weeks as seen in $95 \%$ of women [6,7]. Women with CTS were more sensitive than men regarding reporting their symptoms [8]. There is a lot of non invasive approach for the treatment of CTS of pregnant women working in IT sectors. One of the non invasive method is using the wearable device (wearable glove mouse) [11]. In this we are going to use the wearable glove mouse along with the tendon and nerve gliding exercises prescribed by the physiotherapists which improves the condition. 


\section{INTRODUCTION}

Carpal Tunnel Syndrome was first described by Sir James Paget in 1854 but the term was coined by Moeirisch. It is a syndrome of compression neuropathy of median nerve at the wrist. Carpal Tunnel Syndrome results in considerable discomfort and pain, limitation of activities of daily living, loss of sleep and work disability. (Levine et al.,1993). Twenty percent of symptomatic subjects with symptoms of pain, numbness, nocturnal parasthesia and tingling sensation in the hand would be expected to have Carpal tunnel syndrome based on the clinical examination and electro physiologic testing. Carpal Tunnel Syndrome during Pregnancy is a common complication of pregnancy (Heckman\& Sassard, 1994). The path physiology of pregnancy related Carpal Tunnel Syndrome (PRCTS) has been mostly attributed to redistribution of fluids (Ekman-Ordeberg et al., 1987; Wand, 1990; Pauda et al.,2001).The signs and symptoms includes Pain that shoots from the hand up the arm as far as the shoulder, tingling in the hands during the day or the night that disrupts sleep and limits the ability to grasp objects with the hands, Weak feeling in the hands, and the inability to pick up small objects, the feeling that the hands are swollen, even if they do not appear to be so, burning numbness and tingling sensation in the thumb and first three fingers, weakness in the muscle at the base of the thumb, near the palm. Motor weakness will be seen in abductor pollicis brevis, flexor polices brevis and opponens pollicis brevis. Passive flexion or hyperextension of the affected hand at the wrist for more than one minute may worsen symptoms.(Phalen.G.S.,1966). Percussion of the median nerve at the wrist causes pares thesis of the digits (Steward.J.D.,1978).

\section{HYPOTHESIS OF THE STUDY}

\section{NULL HYPOTHESIS}

There will be no beneficial effect of non invasive solution of using the wearable device along with the tendon and nerve gliding exercises prescribed by the physiotherapist for the relief of symptoms of Carpal tunnel syndrome during pregnancy period of IT women professionals.

\section{ALTERNATE HYPOTHESIS}

There will be beneficial effect of non invasive solution of using the wearable device along with the tendon and nerve gliding exercises prescribed by the physiotherapist for the relief of symptoms of Carpal tunnel syndrome during pregnancy period of IT women professionals.

\section{METHODOLOGY}

Study design: Quasi Experimental Study

Study Setting: Shri Indra Ganesan Institute of Medical Science Physical Medicine and Rehabilitation, Trichirappalli

Sampling method: Convenient sampling - method 
Sample size: According to prevalence rate required sample size is four participants. Each group there are two participants. The convenient samples of four subjects from the middle aged women will be taken for this study. The subjects are selection form the following criteria.

\section{INCLUSION CRITERIA}

- Age group between 21 to 35

- Females

- Pregnancy of third trimester

- Pregnant women with pain and numbness in the wrist, fingers

- Pregnant women with tingling sensation in the wrist, fingers

- Positive Phalen's test

- Nocturnal parasthesia in wrist and fingers

\section{EXCLUSION CRITERIA}

- Non Pregnant women

- Inflammatory joint disease,

- Wrist bone fractures

- Hand surgery

- Age above 35 years

- Multiparous women

- Any other Wrist abnormalities including, diabetes, gout, autoimmune disease

- Persons with rheumatoid arthritis

- Malignancy at wrist

- Peripheral Neuropathy

\section{OUT COME MEASURES}

Visual Analogue Scale and Functional Status Scale.

\section{PROCEDURE}

The Randomised Controlled Trait (RCT) carried in the Department of Physical Medicine and Rehabilitation. Pregnant Women who are working in IT, who are diagnosed with Carpal Tunnel syndrome and are interested in study were invited to undergo Physical examinations. After the confirmation of the CTS with the help of positive Tinel's sign and positive Phalen's test they were separated into two groups. We have to measure the individual changes. Two subjects were selected for experimental group who accepts for wearing the glove mouse during her system work along with doing the tendon and nerve gliding exercises. The control group does only exercise program ( tendon and nerve gliding exercises). The pregnant women were studied only during the third trimester. The duration of the work is four weeks. 
Both the group were given the exercise programmes. The duration of exercise programme is 10 minutes every three to five times per day.

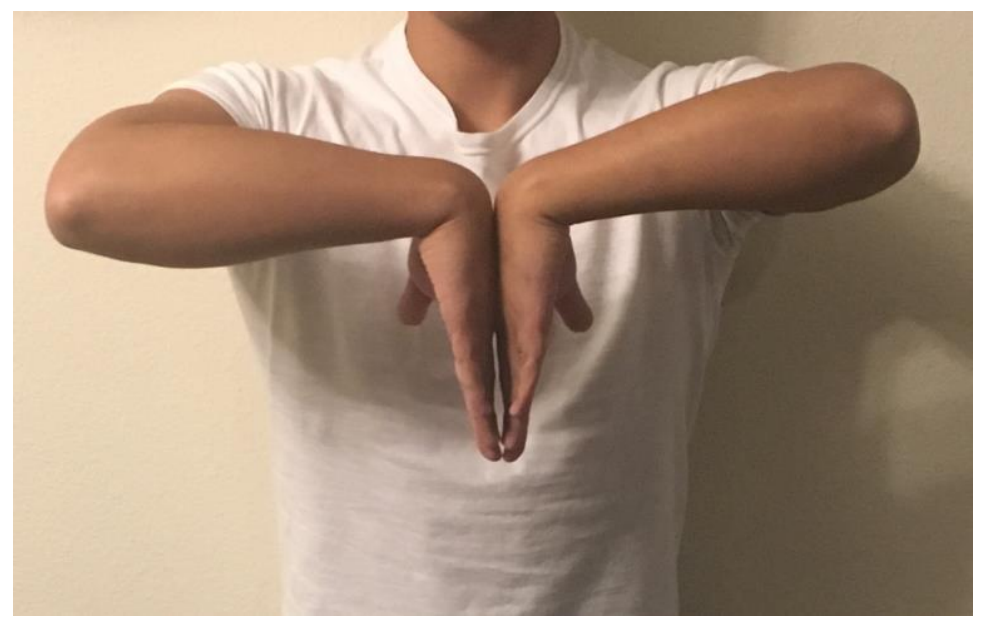

Fig. 1.1. Phalen's Test

Tendon Gliding Exercises / Fist Flexion Exercises: These exercises are thought to relieve pressure on the median nerve and stretch the carpal ligaments, which also helps decrease pressure. They are also thought to help blood flow out of the carpal tunnel, which can help decrease fluid pressure. We have to move our fingers through five positions while our wrist stays in a neutral position(meaning it is not bend). To perform this exercise, we have to do the following:

1. Start with your fingers straight.

2. Make a hook fist and then return to a straight hand.

3. Make a straight fist and then return to a straight hand.

4. Make a full fist and then return to a straight hand.

Hold each position for seven seconds and do 10 repetitions. Repeat three times to five times a day.

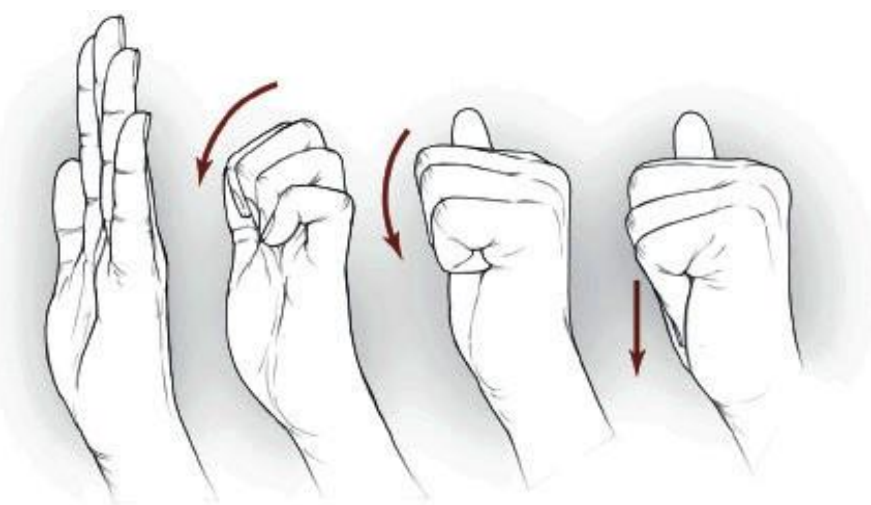

Straight hand Hook fist Full fist Straight fist

Fig.1.2. Tendon Gliding Exercises 
Median Nerve Gliding Exercises: For median nerve gliding exercises we must move our thumb through 6 positions while our wrist stays a neutral position. To perform this exercise, we have do the following:

1. Begin by making a fist with your wrist in the neutral position.

2. Straigthen your fingers and thumb.

3. Bend your wrist back and move your thumb away from your palm

4. Turn your wrist palm up

5. Use your other hand to gently pull out thumb farther away from your palm.

Hold each position for seven seconds, and do five repetitions. Repeat three to five times a day. Effectiveness of the Tendon and nerve gliding exercises used as conservative treatment approaches in relieving the symptoms of the carpal tunnel syndrome during pregnancy.(Lamia Pinar, Asgel Enhos et al.,)

1.
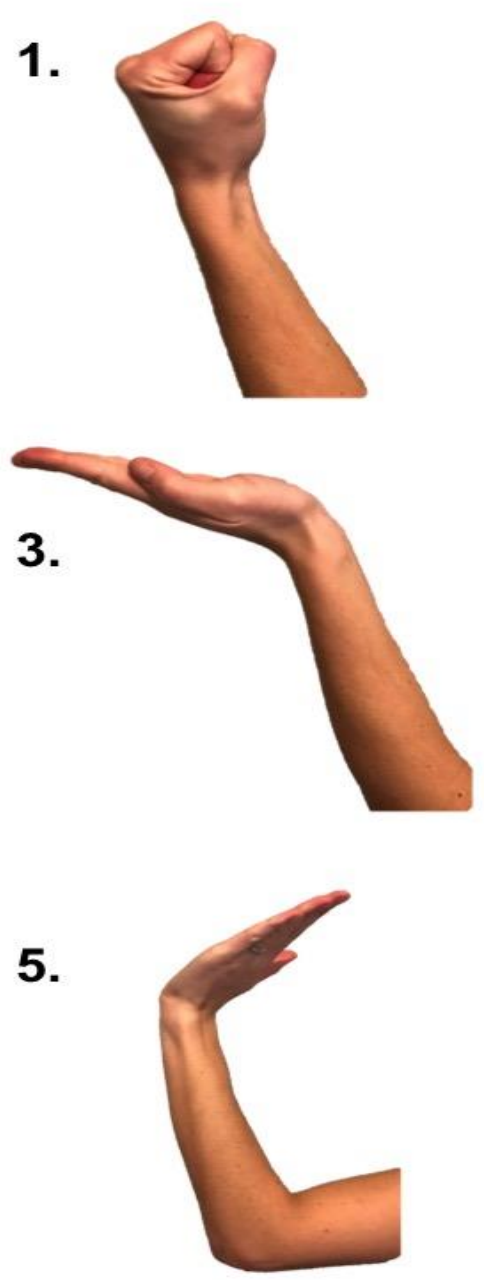

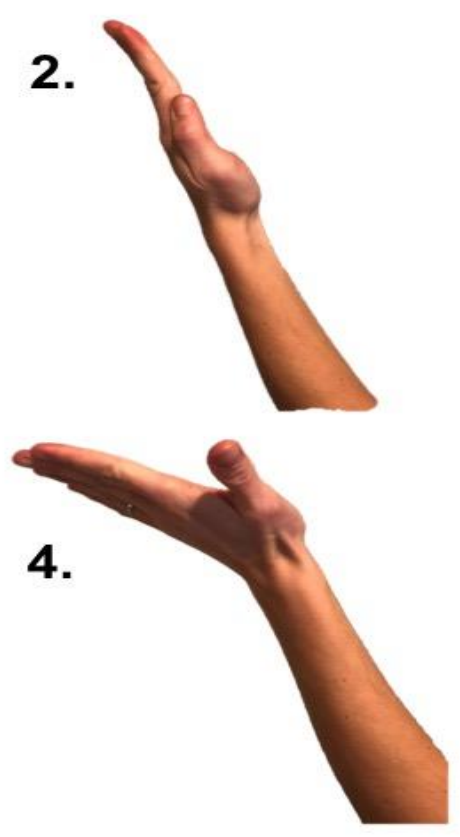

6.

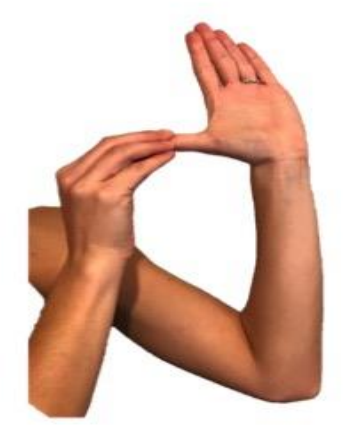

Fig. 1.3. Nerve Gliding Exercises 


\section{Wearable Glove Mouse}

Wearable computer mouse shown in Fig 1.4 can be used with any computer. Wearable glove mouse will work as an alternative to conventional mouse. It focuses more on the step taken to prevent carpal tunnel syndrome. Wearable unit will comprise of flex sensors mounted on glove and centre finger for clicks drag and drop, axis detecting device accelerometer over the glove for detecting the movement and getting the heading of movement. Afterwards the microcontroller will convert movements that is analogue signals to digital signals Flex sensors are utilized for empowering and handicapping the mouse.
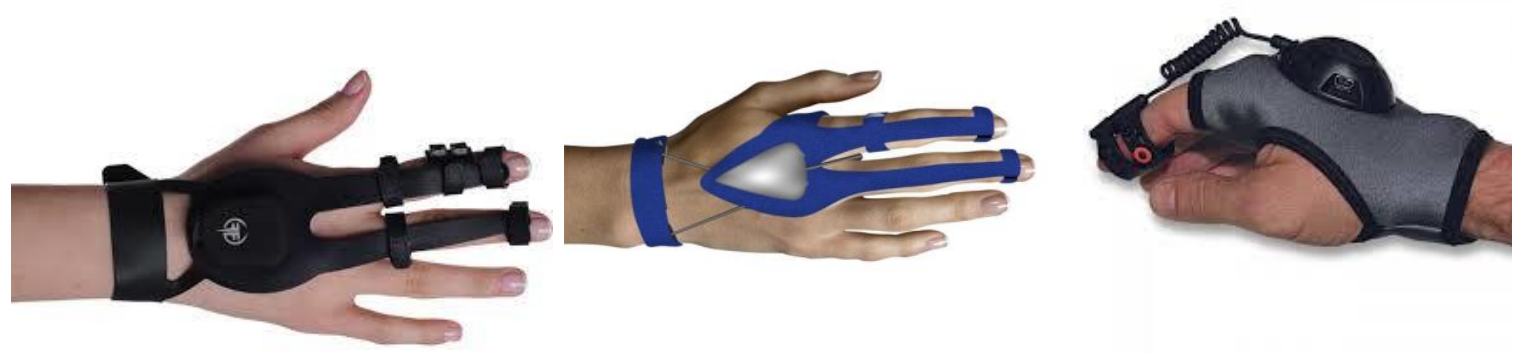

Fig. 1.4. Wearable Glove Mouse

\section{Visual Analogue Scale (VAS)}

The VAS Scale is the valid and reliable measurement tool for pain. It is used to measure the pain response that the patient experience before and after nerve and tendon gliding exercises of median nerve. VAS Scale consists of $10 \mathrm{~cm}$ horizontal line with two ends labelled as no pain(0) and severe pain(10), the patient will mark a point on the line, which corresponds to the intensity of pain what they experience.

\section{Functional Status Scale (FSS)}

Functional Status Scale was used to measure the functional disability offhand. It consists of six items of questionnaire (writing, buttoning of clothes, holding a book while reading, household, carrying grocery bags, bathing and dressing).

Scoring : 06-12 -No difficulty, 13-18 -Mild difficulty, 19-24 -Moderate difficulty, 25-29 cannot do at all due to hand or wrist symptoms. 


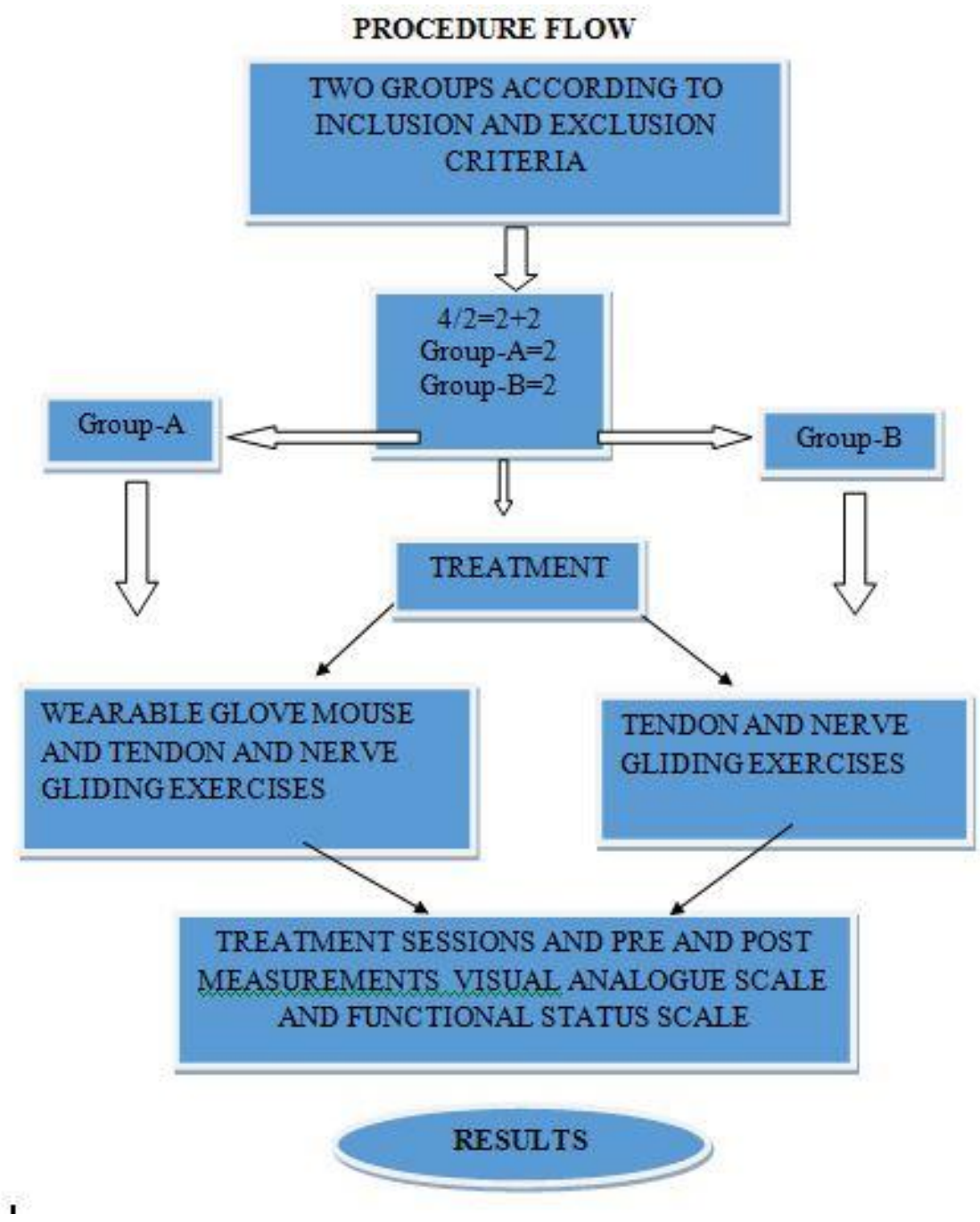

Fig. : 1.5 Procedure for Data collection method

Procedure: A total of 2 subjects with symptomatic carpal tunnel syndrome during pregnancy working in IT field were selected randomly with due consideration to inclusion an exclusion criteria. A brief demonstration was given about tendon and nerve gliding techniques for both the experimental and control group. The subjects were given nerve and tendon gliding exercises. The experimental group were alone given the wearable glove mouse to use while work. The Pre test data and post test data was collected with VAS and FSS Scale. The results were recorded. The results of post test of the same parameter was recorded and compared.

Table -1 . Pre test -Post test values of group $-\mathrm{A}$

\begin{tabular}{|c|c|c|c|c|}
\hline \multirow{2}{*}{ Group A } & \multicolumn{2}{|c|}{ Pre- Value } & \multicolumn{2}{c|}{ Post- value } \\
\cline { 2 - 5 } & Mean & SD & Mean & SD \\
\hline VAS & 7.8 & 0.74 & 1.0 & 0.1 \\
\hline FSS & 25 & 1.41 & 12.6 & 1.01 \\
\hline
\end{tabular}


Table -2Pre test-Post test values of group -B

\begin{tabular}{|c|c|c|c|c|}
\hline \multirow{2}{*}{ Group B } & \multicolumn{2}{|c|}{ Pre- Value } & \multicolumn{2}{c|}{ Post- value } \\
\cline { 2 - 5 } & Mean & SD & Mean & SD \\
\hline VAS & 8.2 & 0.77 & 6.26 & .96 \\
\hline FSS & 25 & 1.41 & 20.6 & 1.8 \\
\hline
\end{tabular}

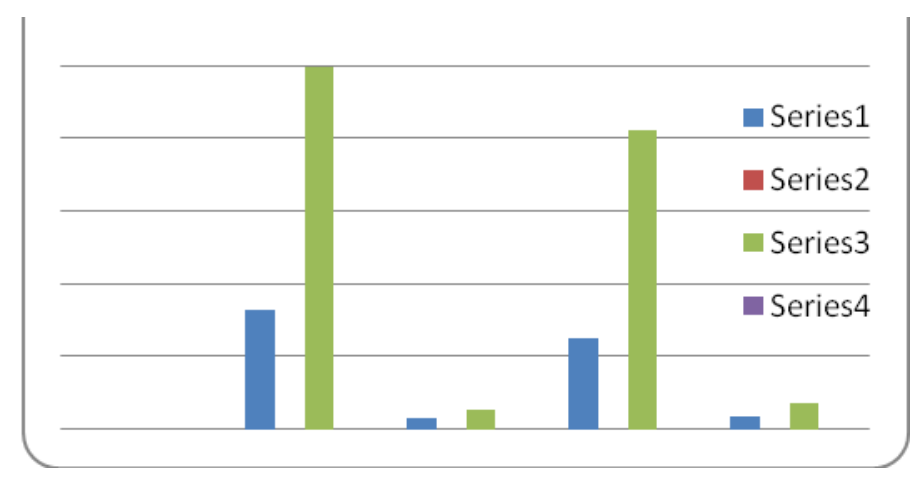

Fig. 1.6 GROUP A

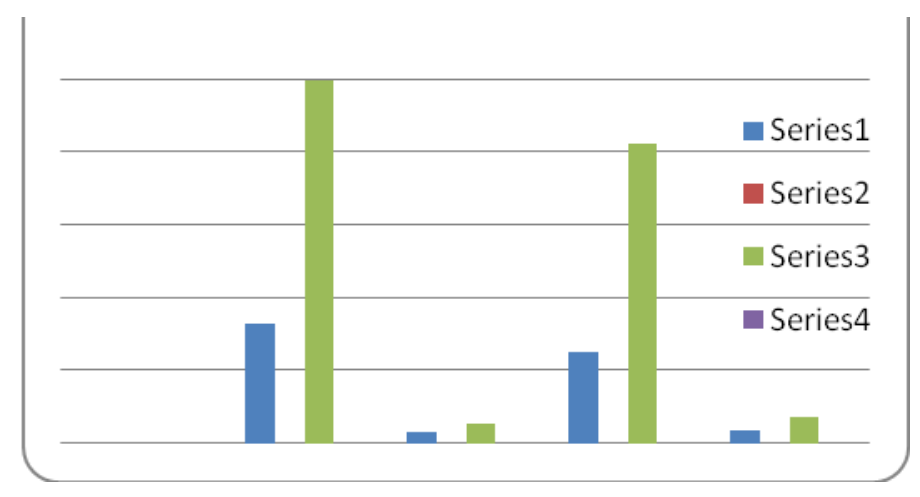

Fig 1.7 GROUP B

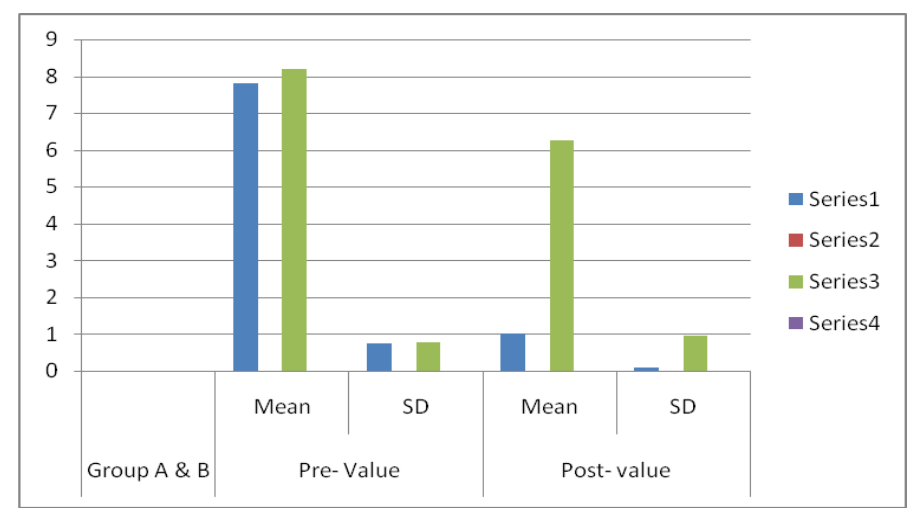

Fig 1.8.Pre and post values of VAS 


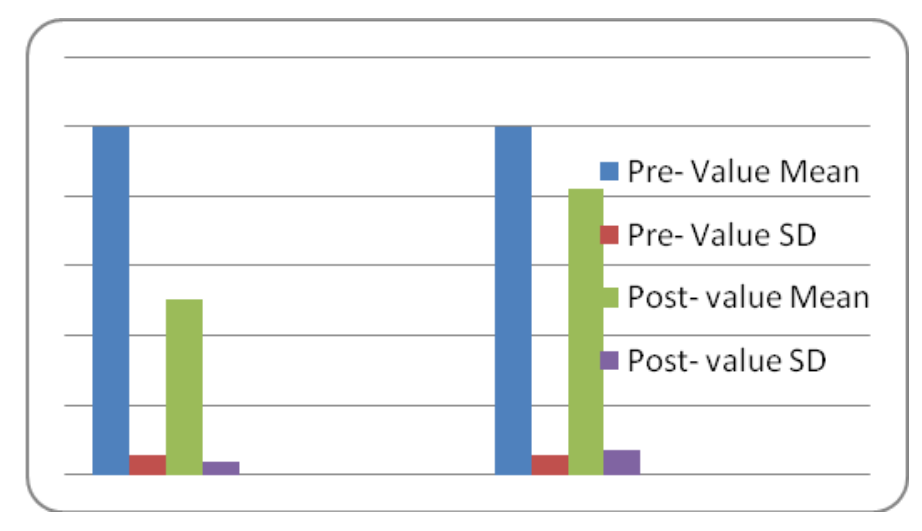

Fig 1.9 Pre and post values of FSS

Table - 3 Comparison between the post test values group A and group B.

\begin{tabular}{|l|l|l|l|l|l|l|}
\hline & \multicolumn{2}{|l|}{ Group A } & \multicolumn{2}{l|}{ Group B } & \multirow{2}{*}{ P-Value } & T -Value \\
\cline { 2 - 6 } & Mean & SD & Mean & SD & & \\
\hline VAS & 1.0 & 0.1 & 6.26 & 0.96 & $<0.0001$ & -12.186 \\
\hline FSS & 12.6 & 1.01 & 20.6 & 1.8 & $<0.0001$ & 8.667 \\
\hline
\end{tabular}

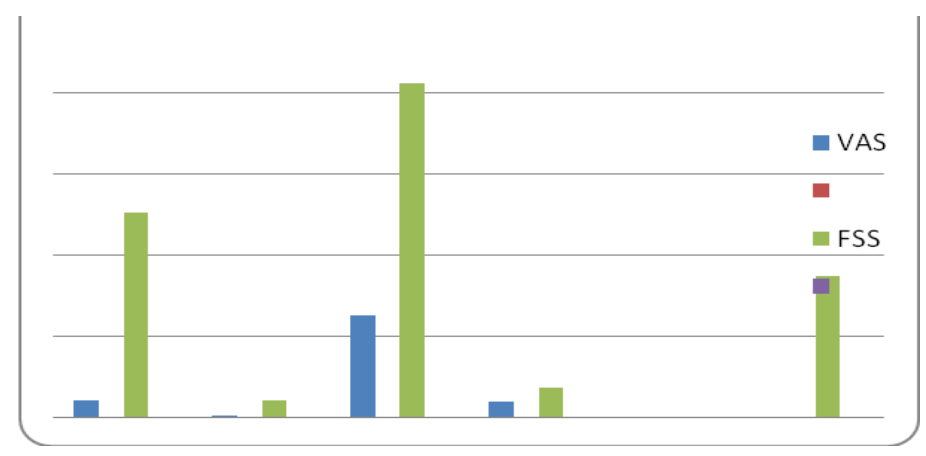

Fig 1.10 Comparison between the post test values group A and group B.

\section{DISCUSSIONS}

Carpal tunnel syndrome is a common condition among people who do repetitive work all day like punching keys on computer or cash register or even pressing on the strings of a violin. Pregnant women are also susceptible to carpal tunnel syndrome even if they do not type or play music all day. The studies have shown that one out of four pregnant women complains of the syndrome usually in the second or third trimester of pregnancy when fluid retention in the arms and hands is more likely to put pressure on the nerve that leads to the hands and fingers. The VAS Scale for pain response and the FSS scale for functional ability which are shown in table 1 and table 2 and in the figure 1.6 and 1.7 respectively shows the effectiveness of the wearable glove mouse given along with the tendon and nerve gliding 
exercises are measured in two groups the experimental group and control group. The former using wearable glove mouse with exercises gives good responses than the later.

In the comparison of pre and post values the two-tailed $\mathrm{P}$ value isless than 0.0001 , by conventional criteria, this difference is considered to be extremely statistically significant. A significance value of $\mathrm{P}$ value and the Confidence interval of the mean of Group One minus Group Two equals -5.260 , 95\% confidence interval of this difference is from -6.2554 to 4.2646and the value of $\mathrm{t}=-12.186, \mathrm{df}=8$, standard error of difference $=0.432$.

On comparing both the groups of FSS results of thetwo-tailed P value is less than 0.0001 by conventional criteria, this difference is considered to be extremely statistically significant. The Confidence interval of the mean of Group One minus Group Two equals 8.000, 95\% confidence interval of this difference: From 5.8714 to 10.1286 and the value of $\mathrm{t}=8.667, \mathrm{df}=8$, standard error of difference $=0.923$.In the software workers who are pregnant the carpal tunnel syndrome during their pregnancy gives more stress and complications. So the results states that use of wearable glove mouse device along with the nerve and tendon gliding exercise reduces the pain and discomfort and improve their functional ability.

\section{CONCLUSION}

The aim of the study was to determine the effect of wearable glove mouse along with tendon and nerve gliding exercises in reducing the pain and improving the functional ability of hand in patient with carpal tunnel syndrome during pregnancy. Carpal tunnel syndrome is most common and significant of all nerve entrapment syndromes (Phalen.G.S. 1972). It causes $20 \%$ of all compression syndromes especially in the third trimester of pregnancies. According to Butler neural tissue requires movement to promote healing and restoration of optimum mechanical properties. Decreased neural mobility and adverse mechanical tension in nervous system can generate pain.(Butler and Elvey 1978). The nervous system is normally under some pressure and tension. If the tension is broke then there results some movement (Millesi.et.al.1972). According to Asgel Enhos tendon requires movement to promote functional ability. The mechanical response is tendon movement in the cross sectional shape and viscous elastic function. So the treatment of tendon Gliding Exercise normalizes the pressure gradient around the nervous system and releases the tendon thus reducing pain improving functional ability. It is found that the static keyboard and mouse are having many limitations so the wearable glove can be used for the same purpose without any limitations. The degree of freedom of glove is more than mouse resulting better inputs in the world of virtualization. So this reduces the pain and difficulties of the carpal tunnel syndrome of the IT ladies who are performing the exercises along with the wearable glove mouse. If the pregnant ladies working with computers started to use the wearable glove mouse along with the home exercises of the tendon and nerve gliding then there will be a considerable reduce in the reoccurrence of the CTS in her post partum period. 


\section{LIMITATIONS AND FUTURE RECOMMENDATIONS}

- Sample size

- Time constraints

- A study with large sample size is recommended

- Further studies shall be done with increased duration of treatment session

- A study shall be done with different outcome measures

\section{REFERENCES}

1. Stolp-Smith KA, Pascoe MK, Ogburn PL. Carpal tunnel syndrome in pregnancy: Frequency, severity, and prognosis. Arch Phys Med Rehabil. 1998 Oct;79(10):1285-7

2. Padua L, Pasquale AD, Pazzaglia C, Liotta GA, Librante A, Mondelli M. Systematic review of pregnancy-related carpal tunnel syndrome. Muscle \& nerve. 2010 Nov; 42(5):697-702.

3. de Oliveira GA, Bernardes JM, de Souza Santos E, Dias A. Carpal tunnel syndrome during the third trimester of pregnancy: prevalence and risk factors. Archives of gynecology and obstetrics. 2019 Sep 1; 300(3):623-31.

4. Klein A. Peripheral Nerve Disease in Pregnancy. Clin Obstet Gynecol. 2013 Jun;56(2):382.

5. Wand JS. Carpal tunnel syndrome in pregnancy and lactation. J Hand Surg Br Eur 1990 Feb 1; 15 (1):93-5.

6. Marsh, M, Glob. libr. women's med., The Nervous System In Pregnancy (ISSN: 17562228) 2008; DOI 10.3843/GLOWM.10166

7.Ekman Ordbeig G (1987) Carpal Tunnel Syndrome in pregnancy. Acta Obsteterica Gynectologicaa Scabdinavy, 66(31), 235-237.

8. A Review of Carpal Tunnel Syndrome and Its Association with Age, Body Mass Index, Cardiovascular Risk Factors, Hand Dominance, and Sex Melissa Airem Cazares-Manríquez. 1, Claudia Camargo Wilson 1, Ricardo Vardasca 2,3,4 , Jorge Luis García-Alcaraz 5, *, Jesús Everardo Olguín-Tiznado 1 , Juan Andrés López-Barreras 6 and Blanca Rosa GarcíaRivera Appl. Sci. 2020, 10, 3488; doi:10.3390/app10103488

9. Hand Data Glove: A Wearable Real-Time Device for HumanComputer Interaction Piyush Kumar1, Jyoti Verma2 and Shitala Prasad3 International Journal of Advanced Science and Technology Vol. 43, June, 2012 
10. Device Development for Detecting Thumb Opposition Impairment Using Carbon Nanotube-Based Strain Sensors Tomoyuki Kuroiwa 1, Akimoto Nimura 2, Yu Takahashi 3 , Toru Sasaki 1 , Takafumi Koyama 1, Atsushi Okawa 1 and Koji Fujita 2, Sensors 2020, 20, 3998; doi:10.3390/s20143998

11. Wearable Device to Prevent Carpel Tunnel Syndrome Inderpreet Singh Vohra,Prabakaran $N *$ and Kannadasan.R International Journal of Pure and Applied Mathematics Volume 119 No. 7 2018, 317-322 ISSN: 1311-8080 (printed version); ISSN: 1314-3395

12. Musculoskeletal considerations in pregnancy. Heckman, J D; Sassard, RThe Journal of Bone \& Joint Surgery: Nov 1994 - Volume 76 - Issue 11 - p 1720-1730 